\title{
Tip Charge Dependence of Three-Dimensional AFM Mapping of Concentrated Ionic Solutions
}

\author{
Simone Benaglia $\odot,{ }^{1}$ Manuel R. Uhlig $\odot,{ }^{1}$ Jose Hernández-Muñoz $\odot,{ }^{2}$ Enrique Chacón®, ${ }^{1}$ \\ Pedro Tarazona, ${ }^{2}$ and Ricardo Garcia ${ }^{1, *}$ \\ ${ }^{1}$ Instituto de Ciencia de Materiales de Madrid, Consejo Superior de Investigaciones Científicas, Madrid 28049, Spain \\ ${ }^{2}$ Departamento de Física Teórica de la Materia Condensada, IFIMAC Condensed Matter Physics Center, \\ Universidad Autónoma de Madrid, Madrid 28049, Spain
}

(Received 21 May 2021; accepted 5 October 2021; published 1 November 2021)

\begin{abstract}
A molecular scale understanding of the organization and structure of a liquid near a solid surface is currently a major challenge in surface science. It has implications across different fields from electrochemistry and energy storage to molecular biology. Three-dimensional AFM generates atomically resolved maps of solid-liquid interfaces. The imaging mechanism behind those maps is under debate, in particular, for concentrated ionic solutions. Theory predicts that the observed contrast should depend on the tip's charged state. Here, by using neutrally, negatively, and positively charged tips, we demonstrate that the 3D maps depend on the tip's polarization. A neutral tip will explore the total particle density distribution (water and ions) while a charged tip will reveal the charge density distribution. The experimental data reproduce the key findings of the theory.
\end{abstract}

DOI: 10.1103/PhysRevLett.127.196101

An atomic-scale understanding of the structure of a solid-liquid interface is of paramount relevance in materials science and biology [1-3]. It has implications in topics as diverse as energy conversion and storage, corrosion, friction, or molecular recognition. For several decades, density functional theories were proposed to describe solidliquid interfaces. The theoretical predictions were commonly tested with computer simulations. Comparisons between experiments and theory for realistic solid-liquid interfaces were scarce because the experimental data did not have atomic-scale features. Therefore, we still lack a molecular-level understanding of the interactions that take place at the solid-liquid interface.

Recently, three-dimensional AFM (3D AFM) [4-7] has provided atomically resolved images of solid-liquid interfaces. This method has revealed the interfacial liquid structure of a variety of hydrophilic surfaces (mica, calcite, boehmite, fluorite, ionic crystals) [7-11] and hydrophobic surfaces such as graphite [12-16], graphene, and other 2D materials surfaces [17]. The interfacial water structure on graphitelike surfaces is under debate, with interpretations ranging from the formation of gas layers $[13,14]$, hydrocarbon layers [16,17], or oriented water molecules [18]. Atomically resolved 3D maps demonstrated the existence of a layer of hydrocarbon molecules (1-2 $\mathrm{nm}$ in thickness) separating the graphite from the water [16]. The formation of this hydrocarbon layer is driven by free energy considerations [19]. It has been proposed that it deteriorates the performance of supercapacitors based on graphite electrodes [20]. Three-dimensional AFM has been applied to image the local water density distribution on proteins [6],
DNA [21,22], lipid bilayers [23], and nanoparticles [24]. The robustness of the method has enabled the 3D molecular mapping of the interfacial structure of organic solvents [25-29], concentrated electrolyte solutions [30], and ionic liquids [31-33]. In particular, it provided high spatial resolution images of the electric double layer of an ionic liquid on an electrode surface [32].

Our current understanding of the imaging mechanism that operates in 3D AFM is based on models and observations performed on dilute or relatively dilute electrolyte solutions $(\leq 1 M)$. It is accepted that the peaks observed in a force-distance profile arise from the interaction of the hydration shell structure at the tip's apex with the hydration structure near the solid surface [34-38]. However, that interpretation might break down in solutions with a significant presence of ionic species [30]. In addition, it does not apply for experiments performed with nonaqueous solutions. For dilute aqueous solutions, molecular dynamics simulations have shown that the tip charge modifies the hydration shell structure at its apex which might eventually affect the atomic-scale imaging [38]. However, there are no studies on the influence of the tip charge on the mapping of the interfacial liquid structure of concentrated ionic solutions. In particular, we hypothesize that the tip charge controls the contrast obtained in 3D AFM experiments performed in concentrated ionic solutions.

This contribution was motivated by the experiments associated with the emerging method of 3D AFM, however, its conclusions apply to the interpretation of experiments performed on ionic liquids by using conventional AFM imaging or force-distance curve methods [39-43]. 
In this Letter, we study the dependence of the tip's charged state in the molecular contrast observed in 3D AFM. The experiments were performed on a concentrated electrolyte solution near a mica surface by using neutrally, positively, and negatively charged tips. Density functional theory (DFT) calculations predict that a neutral tip will explore the total particle density distribution (water and ions). On the other hand, a charged tip will reveal the local charge density distribution across the interface. Threedimensional AFM experiments performed on a concentrated $\mathrm{NaCl}$ solution reveal major differences in the observed interfacial liquid structure as a function of the charged state of the tip. The force maps acquired with a neutral tip reflect the particle density distribution while the force maps obtained by using a charged tip are associated with the charge density distribution across the interface. The experimental results reproduce a key theoretical prediction regarding the shift of the peak force dependence on the tip-surface distance as a function of the tip's polarity. At the distance where a positive tip detects a local peak force value (repulsive force), a negative tip will show a local minimum in the force (attractive). Therefore, the observed contrast is dominated by electrostatic interactions if the tip is charged.

The interface of a concentrated electrolyte solution near a mica surface is characterized by the alternation of cation and anion layers with a significant amount of water in between [30]. This interface is very stable in the presence of an AFM tip. It does not degrade the geometry nor chemical properties of the tip. We propose this interface as a model to test the influence of the tip charge on the interfacial liquid structure observed by 3D AFM. The Supplemental Material provides the information needed to generate concentrated electrolyte solutions on mica [44].

Density functional theory (DFT) calculations for a concentrated $\mathrm{NaCl}$ aqueous solution on a mica surface establish that the interfacial charge density (density of cations minus density of anions) depends on the tip charge [right panels of Fig. 1(a)]. The presence of a tip has little influence on the charge distribution of the ions on or near the mica (angstroms range), however, it introduces significant changes in the charge distribution around the tip's apex (Supplemental Material [44]) which, in turn, will modify the tip-surface forces at a given separation. A positive tip draws an atomic size layer of anions toward it while an atomic size layer of cations will be formed on a negative tip. The particle density distribution maps [water + cations $\left(\mathrm{Na}^{+}\right)+$anions $\left.\left(\mathrm{Cl}^{-}\right)\right]$[left panels of Fig. 1(a)], show little variations for neutrally, positively, and negatively charged tips as a function of $z$ for a given tip-mica distance $Z$. The DFT model is described in previous publications [58,59]. Additional details are presented in Supplemental Material [44].

The overlap between the ion layer at the tip's apex and the charge density distribution of the electrolyte is reflected

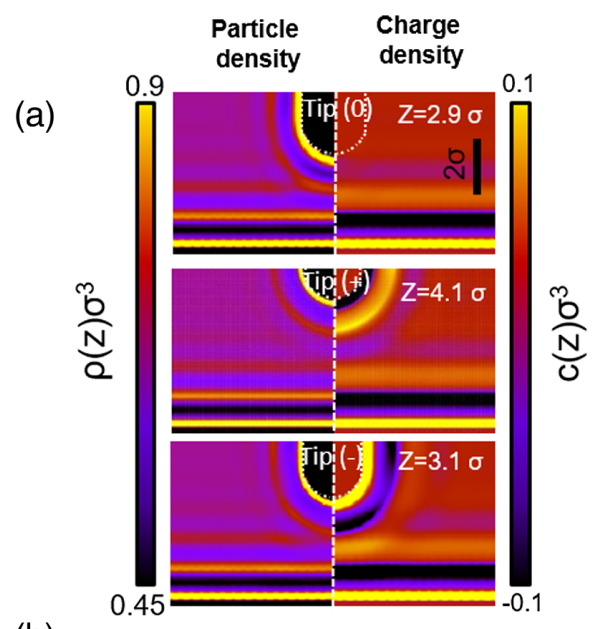

(b)
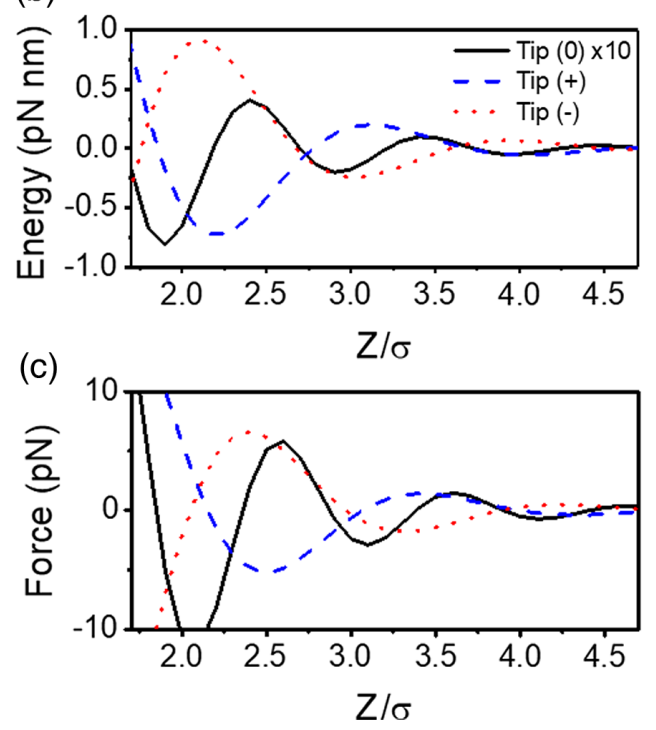

FIG. 1. DFT particle [left, $\rho(z)$ ] and charge density [right, $c(z)$ ] distributions of a concentrated electrolyte solution near a mica surface for different tips. (a) The simulations reproduce a tipsample approach. The sphere-cylindrical tip's contour is marked with a dotted white line. The maps are symmetric with respect to the tip's vertical axis. For that reason and to include particle and charge density distributions in a single plot, we just represent half of the maps. The color-coded scale bar on the left panels represents the particles per $\sigma^{3}$ volume. The color-coded scale bar on right panels represents the total charges per unit volume (positive charges per $\sigma^{3}$ volume-negative charges per $\sigma^{3}$ volume). (b) Free energy profile as a function of the tip-mica distance normalized to the hard sphere diameter $\sigma=0.3166 \mathrm{~nm}$. (c) Force-distance curve derived from (b). Energy and force curves are obtained by fitting values from discrete DFT minimization as described in the Supplemental Material [44]. Note that the force (and energy) distance curve of the neutral tip was multiplied by 10 . Densities of particles and charge are given in dimensionless units. The dimensionless values could be converted into particle per volume and charge per volume values. In the first case, by dividing the values by the cube of the molecular diameter $\sigma^{3}$. In second case, by multiplying the values by the elementary charge of the ions $( \pm e)$ and divinding the result by the cube of the molecular diameter. 
in the free energy profile as a function of the tip-sample distance $Z$ (separation between tip and sample) [Fig. 1(b)]. The free energy profiles show an oscillatory behavior which depends on the tip charge. A similar behavior is observed in the force-distance profile (negative derivative of the free energy with respect to $Z$ at each $x y$ position). The force is the main observable in a 3D AFM experiment. A comparison between particle and charge density distributions shows the dependence of the latter on the tip charge (Supplemental Material [44]). Therefore, we expect the 3D AFM maps of a concentrated ionic solution will depend on the charge of the tip.

To test the theoretical predictions, we performed a series of 3D AFM measurements in a very concentrated $\mathrm{NaCl}$ solution near a mica surface. In the experiments, the molarity of the solution was very close to the saturation value [44]. The experiments were performed by operating the 3D AFM with an amplitude modulation feedback $[30,60]$. We have used three different types of tips, neutrally, positively, and negatively charged. The charge of the tip was controlled by chemical functionalization and the $p \mathrm{H}$ of the solution [Fig. 2(a)].

Figure 2(b) shows three representative 3D AFM maps of a very concentrated $\mathrm{NaCl}$ solution near a mica surface. The contrast reflects the layering of the liquid molecules above the mica. For a neutral tip, the atomic-scale corrugation on the mica surface is also observed. To quantify the differences observed in the layering as a function of the tip charge, we extract some representative 2D $F(x, Z)$ maps from the 3D AFM data [Fig. 2(c)]. The maps show the alternation of layers with relatively high and low force values and their strong dependence on the tip charge. From the corresponding force-distance curves (FDCs) [Fig. 2(d)], we obtain that the distance between the first and second layer $\left(d_{1}\right)$ and the second and the third layer $\left(d_{2}\right)$ for a neutral tip is of 0.24 and $0.32 \mathrm{~nm}$. For a negative tip, the values are, respectively, $d_{1}=0.41 \mathrm{~nm}$ and $d_{2}=0.45 \mathrm{~nm}$. For a positive tip only two layers are observed with $d_{1}=0.50 \mathrm{~nm}$. The dependence of interlayer separations on the tip charge agrees with the DFT simulations. We have performed additional 3D AFM experiments by using other types of uncharged tips such as diamondlike carbon tips (Fig. S2 of Supplemental Material [44]). Those tips gave interlayer distances consistent with the expected values for hydration layers. We conclude that a neutral tip provides force maps that closely follow the particle density distribution. Those maps are characterized by interlayer spacings of about $0.3 \mathrm{~nm}$, this is, identical to the ones given by
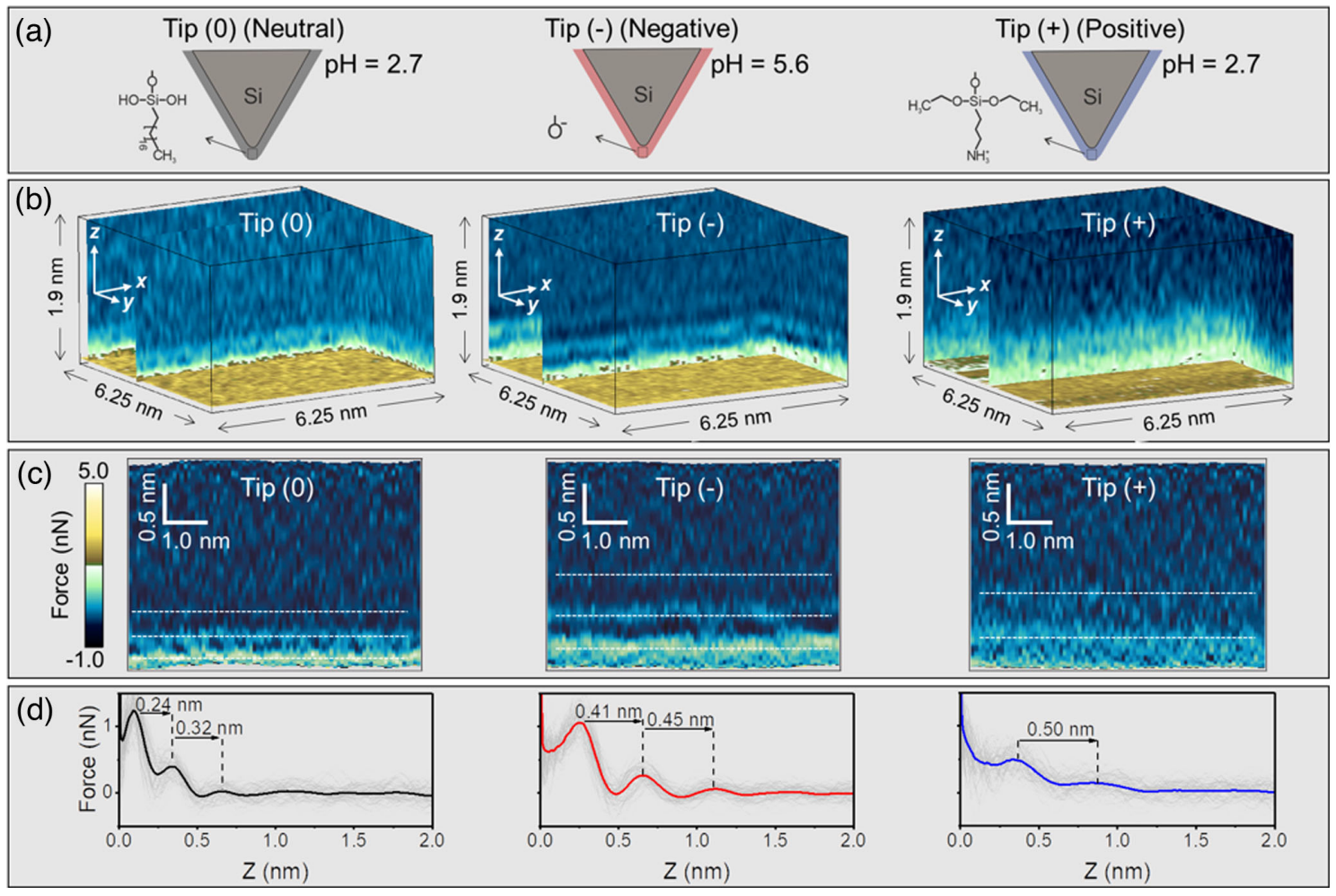

FIG. 2. 3D AFM force maps and scheme of the experimental tips. (a) The tip charge was controlled by chemical functionalization and selecting the $\mathrm{pH}$. (b) 3D AFM maps of a concentrated $\mathrm{NaCl}$ solution acquired with neutral [tip $(0)$ ], negative [tip $(-)$ ], and positive $[\operatorname{tip}(+)]$ tips depicted in (a). The above maps show variations of the phase shift $\phi(x, y, Z)$ as a function of the tip's position. Force reconstruction methods enable us to transform phase shift changes into forces [44]. (c) 2D force $(x, Z)$ extracted for the 3D AFM maps for the different tips. (d) Force-distance curves extracted from the 2D force maps. Individual curves of the map (one for each $x$ position) are plotted in gray. The solid line represents the average FDC. The molarity of the concentrated $\mathrm{NaCl}$ solution was $\sim 5.5 M$. 

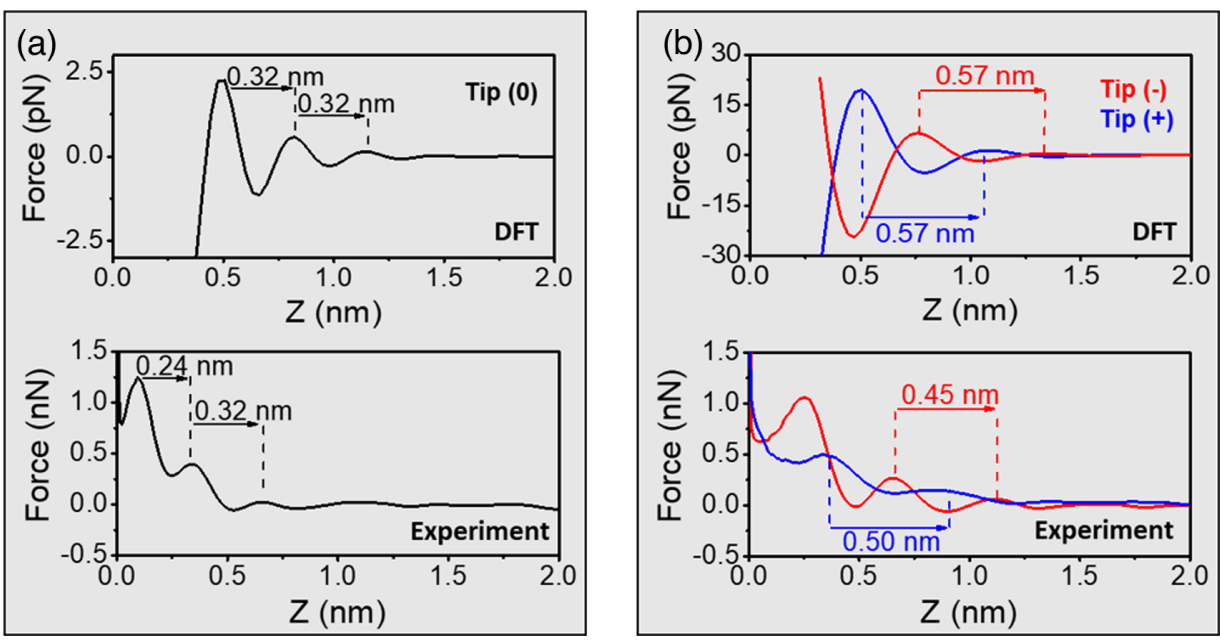

FIG. 3. Comparison between DFT and experimental force-distance curves. (a) FDC obtained through a neutral tip. (b) FDC obtained through a negative (red) and positive (blue) tip. In the experiment, the direct contact between the tip and the mica surface defines $Z=0$. The experiments and the DFT results are for a concentrated $\mathrm{NaCl}$ solution.

hydration layers. On the other hand, a charged tip maps the local charge density distribution which is characterized by larger interlayer distances.

Figure 3 shows the experimental and DFT force-distance curves (average values) obtained with neutrally [Fig. 3(a)], negatively [Fig. 3(b)], and positively [Fig. 3(b)] charged tips. The agreement obtained between the DFT simulations and experiments includes three major points. First, for a neutral tip, the interlayer distances are very similar $(0.24-0.32 \mathrm{~nm}$ range). That range is the expected for the separation between hydration layers on mica [17,30]. Second, the interlayer separations for a charged tip are about $0.2 \mathrm{~nm}$ larger $(\sim 0.5 \mathrm{~nm})$. Third, a change in the sign of the tip's polarity introduces a shift in the $z$ positions of the local maxima and minima of the FDCs. The force profile obtained with a positive tip is offset along the $z$ axis by half of a period with respect to the one obtained with a negative tip. The DFT results show that for a charged tip, the peak and minimum force values are associated with the overlap between the ion layer at the tip's apex and the density distributions of the two ions in the electrolyte. The overlap with the density distribution of the same ion gives a minimum of the grand potential, while the overlap with counterions gives a local maximum in that potential. The force takes maximum and minimum values in between the maxima and minima of the effective potential. The entropy of atomic packing competes with the influence of the substrate and the tip to break the local charge neutrality. For a neutral tip, attractive (repulsive) force peaks are associated with the overlap of the water molecules at the tip's apex with an upper (lower) edge of a surface water density peak. Even for very concentrated electrolyte solutions, the
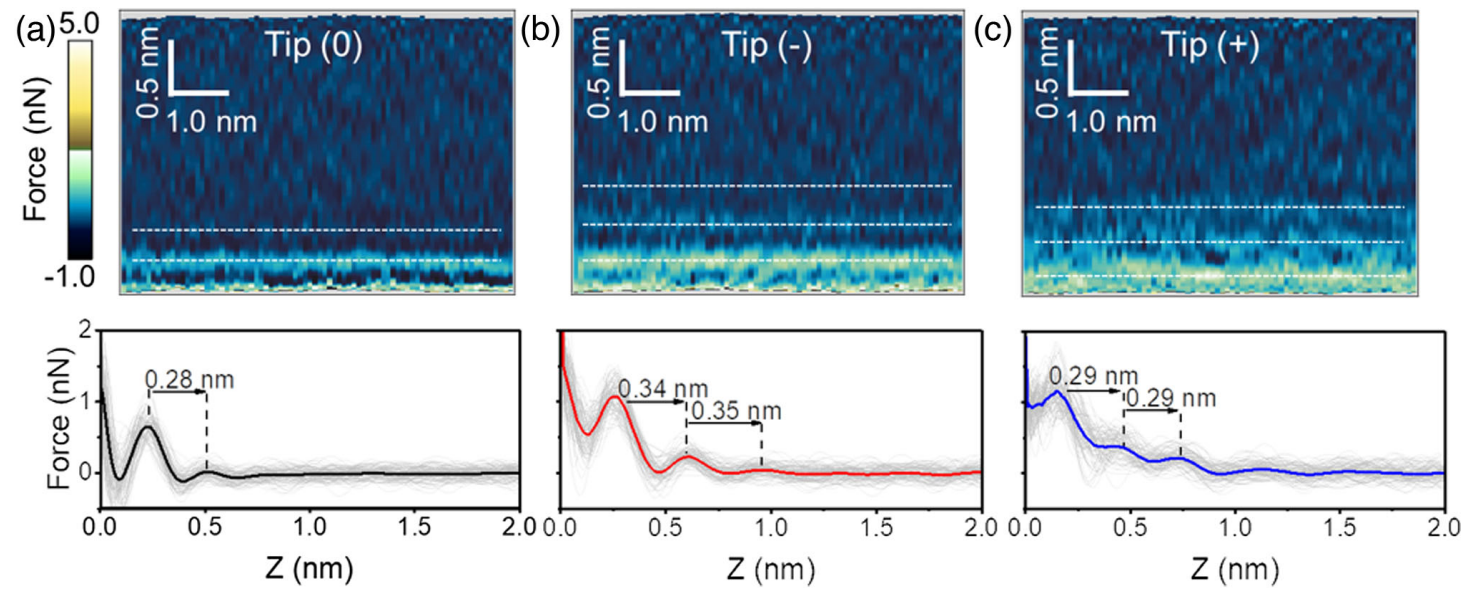

FIG. 4. 2D force maps and force-distance curves for a $1 M \mathrm{NaCl}$ solution near a mica surface. (a) 2D force $(x, Z)$ maps and FDCs by using a neutral tip. (b) 2D force $(x, Z)$ maps and FDCs by using a negative tip. (c) 2D force $(x, Z)$ maps and FDCs by using a positive tip. Curves in gray are individual curves included in the 2D map. The solid line represents the average force-distance curve. 
particle density profile beyond the first adsorbed layer is dominated by the density of the water (see Figs. S7 and S8 of Supplemental Material [44]).

The DFT calculations involve several approximations aimed to capture the essential aspects of the system. The theoretical model departs from the actual experimental systems in several details, it assumes equal atomic radius for all the species and uses simple geometrical forms for substrate and tip. The tip in the model is significantly smaller than in the experiment. Those factors account for the numerical differences observed in values of the DFT and 3D AFM forces. However, the experiment and the theory provide force-distance curves with the same separations ( $Z$ values) of the local maxima and minima. This agreement underlines that the theoretical model reproduces the key aspects of the experimental data.

We have investigated the solid-electrolyte interface obtained by using $\mathrm{NaCl}$ solutions of lower molarities. Figure 4 shows 2D $F(x, Z)$ maps obtained with neutrally, positively and negatively charged tips on a $1 M \mathrm{NaCl}$ solution near a mica surface. The $2 \mathrm{D}$ force maps and the corresponding FDCs show several layers. In all three cases, the interlayer distances are approximately of $0.3 \mathrm{~nm}$. This value coincides with the separation observed between hydration layers on mica $[4,7,16]$. This result demonstrates that for low to moderate molarity solutions $(\leq 1 M)$, the particle density distribution of the interface dominates the observed contrast in 3D AFM. The force profile reflects that the total particle density is dominated by the water. In this case, $\mathrm{Na}+$ ions are adsorbed on the trigonal cavities of the mica surface while the $\mathrm{Cl}-$ ions are expelled from the surface. Thus, the interfacial structure reveals the one of the water with no or little influence by the charge of the tip. This observation is in agreement with MD simulations [34]. Therefore, we conclude that the influence of the tip's polarity on the 3D AFM maps happens for experiments involving solutions with a significant change in the charge density distribution across the interface, such as very concentrated electrolyte solutions and ionic liquids. Experiments performed on a graphite surface immersed in an ionic liquid confirm the above conclusion (see Fig. S5 of Supplemental Material [44]).

The present results were discussed in the context set by 3D AFM, however, the conclusions also apply for other AFM measurements performed in liquid [40,42,43,61-63]. In general, neutral tips are recommended for generating force maps with a close relationship with the particle density distribution. On the other hand, a charged tip can be used to enhance the electrostatic interactions with the charged species of the interface.

In summary, we demonstrate that the contrast mechanism operating in 3D AFM depends on the tip's charged state. On an interface formed by a mica surface and a very concentrated electrolyte solution, a neutral tip will generate 3D maps of the interface that will follow the particle density distribution. On the other hand, a charged tip will map the charge density distribution variations across the interface. In the latter case, electrostatic interactions will dominate the force. As a consequence, the positions of the peaks measured with a positive tip will be offset by about half of the interlayer distance with respect to the positions given by a negative tip.

This work was funded by the Ministerio de Ciencia e Innovación (Spain) under Grants No. PID2019-106801GBI00, No. FIS2017-86007-C3, and No. FPU2015/0248, the "María de Maeztu" Programme for Units of Excellence in R\&D (CEX2018-000805-M) and the CSIC (202050E013).

S. B. performed the experiments. S. B. and M.R.U. analyzed the data. M. R. U. developed the code for 3D AFM data analysis. J. H. M., E. C., and P. T. conceived and performed the DFT calculations. R. G. conceived the 3D AFM method and drafted the manuscript. All authors analyzed and discussed the data, and contributed to the final version of the manuscript.

* Corresponding author. r.garcia@csic.es

[1] O. Björneholm, M. H. Hansen, A. Hodgson, L. M. Liu, D. T. Limmer, A. Michaelides, P. Pedevilla, J. Rossmeisl, H. Shen, G. Tocci, E. Tyrode, M. M. Walz, J. Werner, and H. Bluhm, Chem. Rev. 116, 7698 (2016).

[2] Y. F. Dufrêne, T. Ando, R. Garcia, D. Alsteens, D. MartinezMartin, A. Engel, C. Gerber, and D. J. Müller, Nat. Nanotechnol. 12, 295 (2017).

[3] V. Maurice and P. Marcus, Prog. Mater. Sci. 95, 132 (2018).

[4] T. Fukuma, Y. Ueda, S. Yoshioka, and H. Asakawa, Phys. Rev. Lett. 104, 016101 (2010).

[5] K. Kimura, S. Ido, N. Oyabu, K. Kobayashi, Y. Hirata, T. Imai, and H. Yamada, J. Chem. Phys. 132, 194705 (2010).

[6] E. T. Herruzo, H. Asakawa, T. Fukuma, and R. Garcia, Nanoscale 5, 2678 (2013).

[7] T. Fukuma and R. Garcia, ACS Nano 12, 11785 (2018).

[8] K. Umeda, L. Zivanovic, K. Kobayashi, J. Ritala, H. Kominami, P. Spijker, A. S. Foster, and H. Yamada, Nat. Commun. 8, 2111 (2017).

[9] H. Söngen, B. Reischl, K. Miyata, R. Bechstein, P. Raiteri, A. L. Rohl, J. D. Gale, T. Fukuma, and A. Kühnle, Phys. Rev. Lett. 120, 116101 (2018).

[10] H. Söngen, C. Marutschke, P. Spijker, E. Holmgren, I. Hermes, R. Bechstein, S. Klassen, J. Tracey, A. S. Foster, and A. Kühnle, Langmuir 33, 125 (2017).

[11] E. Nakouzi, A. G. Stack, S. Kerisit, B. A. Legg, C. J. Mundy, G. K. Schenter, J. Chun, and J. J. De Yoreo, J. Phys. Chem. C 125, 1282 (2021).

[12] K. Suzuki, N. Oyabu, K. Kobayashi, K. Matsushige, and H. Yamada, Appl. Phys. Express 4, 125102 (2011).

[13] I. Schlesinger and U. Sivan, J. Am. Chem. Soc. 140, 10473 (2018).

[14] C. W. Yang, K. Miyazawa, T. Fukuma, K. Miyata, and I. S. Hwang, Phys. Chem. Chem. Phys. 20, 23522 (2018). 
[15] H. Teshima, Q.-Y. Li, Y. Takata, and K. Takahashi, Phys. Chem. Chem. Phys. 22, 13629 (2020).

[16] M. R. Uhlig, S. Benaglia, R. Thakkar, J. Comer, and R. Garcia, Nanoscale 13, 5275 (2021).

[17] M. R. Uhlig, D. Martin-Jimenez, and R. Garcia, Nat. Commun. 10, 2606 (2019).

[18] L. Fumagalli, A. Esfandiar, R. Fabregas, S. Hu, P. Ares, A. Janardanan, Q. Yang, B. Radha, T. Taniguchi, K. Watanabe, G. Gomila, K. S. Novoselov, and A. K. Geim, Science 360, 1339 (2018).

[19] E. R. Azhagiya Singam, Y. Zhang, G. Magnin, I. Miranda-Carvajal, L. Coates, R. Thakkar, H. Poblete, and J. Comer, J. Chem. Theory Comput. 15, 1302 (2019).

[20] T. T. Duignan and X. S. Zhao, J. Phys. Chem. C 123, 4085 (2019).

[21] K. Kuchuk and U. Sivan, Nano Lett. 18, 2733 (2018).

[22] K. Umeda, K. Kobayashi, N. Oyabu, K. Matsushige, and H. Yamada, Nanotechnology 26, 285103 (2015).

[23] H. Asakawa, S. Yoshioka, K. Nishimura, and T. Fukuma, ACS Nano 6, 9013 (2012).

[24] P. J. Molino, D. Yang, M. Penna, K. Miyazawa, B. R. Knowles, S. MacLaughlin, T. Fukuma, I. Yarovsky, and M. J. Higgins, ACS Nano 12, 11610 (2018).

[25] P. Spijker, T. Hiasa, T. Musso, R. Nishioka, H. Onishi, and A. S. Foster, J. Phys. Chem. C 118, 2058 (2014).

[26] T. Minato, Y. Araki, K. Umeda, T. Yamanaka, K. Okazaki, H. Onishi, T. Abe, and Z. Ogumi, J. Chem. Phys. 147, 124701 (2017).

[27] Y. Teduka, A. Sasahara, and H. Onishi, ACS Omega 5, 2569 (2020).

[28] J. Hernández-Muñoz, M. R. Uhlig, S. Benaglia, E. Chacón, P. Tarazona, and R. Garcia, J. Phys. Chem. C 124, 26296 (2020).

[29] W. Foster, K. Miyazawa, T. Fukuma, H. Kusumaatmaja, and K. Voïtchovsky, Nanoscale 12, 5452 (2020).

[30] D. Martin-Jimenez, E. Chacon, P. Tarazona, and R. Garcia, Nat. Commun. 7, 12164 (2016).

[31] K. Umeda, K. Kobayashi, T. Minato, and H. Yamada, J. Phys. Chem. Lett. 11, 1343 (2020).

[32] S. Zhou, K. S. Panse, M. H. Motevaselian, N. R. Aluru, and Y. Zhang, ACS Nano 14, 17515 (2020).

[33] G. Krämer and R. Bennewitz, J. Phys. Chem. C 123, 28284 (2019).

[34] M. Watkins, M. L. Berkowitz, and A. L. Shluger, Phys. Chem. Chem. Phys. 13, 12584 (2011).

[35] M. Watkins and B. Reischl, J. Chem. Phys. 138, 154703 (2013).

[36] K. Miyazawa, N. Kobayashi, M. Watkins, A. L. Shluger, K. Amano, and T. Fukuma, Nanoscale 8, 7334 (2016).

[37] T. Fukuma, B. Reischl, N. Kobayashi, P. Spijker, F. F. Canova, K. Miyazawa, and A. S. Foster, Phys. Rev. B 92, 155412 (2015).

[38] K. Miyazawa, J. Tracey, B. Reischl, P. Spijker, A. S. Foster, A. L. Rohl, and T. Fukuma, Nanoscale 12, 12856 (2020).

[39] R. Atkin and G. G. Warr, J. Phys. Chem. C 111, 5162 (2007).

[40] J. M. Black, D. Walters, A. Labuda, G. Feng, P. C. Hillesheim, S. Dai, P. T. Cummings, S. V. Kalinin, R. Proksch, and N. Balke, Nano Lett. 13, 5954 (2013).
[41] A. Elbourne, S. McDonald, K. Voïchovsky, F. Endres, G. G. Warr, and R. Atkin, ACS Nano 9, 7608 (2015).

[42] J. M. Black, M. Zhu, P. Zhang, R. R. Unocic, D. Guo, M. B. Okatan, S. Dai, P. T. Cummings, S. V. Kalinin, G. Feng, and N. Balke, Sci. Rep. 6, 32389 (2016).

[43] D. A. Rakov, F. Chen, S. A. Ferdousi, H. Li, T. Pathirana, A. N. Simonov, P. C. Howlett, R. Atkin, and M. Forsyth, Nat. Mater. 19, 1096 (2020).

[44] See Supplemental Material at http://link.aps.org/ supplemental/10.1103/PhysRevLett.127.196101 for additional experimental and theoretical data, i.e., details about 3D AFM experiments, measurement conditions, experimental 3D panels obtained with additional tips, description of DFT calculations, plots of the simulated density profiles, and which includes Refs. [45-57].

[45] J. E. Sader and J. R. Friend, Rev. Sci. Instrum. 85, 116101 (2014).

[46] J. E. Sader and J. R. Friend, Rev. Sci. Instrum. 86, 056106 (2015).

[47] A. Labuda, M. Kocun, M. Lysy, T. Walsh, J. Meinhold, T. Proksch, W. Meinhold, C. Anderson, and R. Proksch, Rev. Sci. Instrum. 87, 073705 (2016).

[48] R. Garcia, Amplitude Modulation Atomic Force Microscopy (Wiley-VCH Verlag GmbH \& Co. KGaA, Weinheim, Germany, 2010).

[49] A. F. Payam, D. Martin-Jimenez, and R. Garcia, Nanotechnology 26, 185706 (2015).

[50] H. Hölscher, Appl. Phys. Lett. 89, 123109 (2006).

[51] J. J. Shyue, M. R. De Guire, T. Nakanishi, Y. Masuda, K. Koumoto, and C. N. Sukenik, Langmuir 20, 8693 (2004).

[52] M. Valtiner, X. Banquy, K. Kristiansen, G. W. Greene, and J. N. Israelachvili, Langmuir 28, 13080 (2012).

[53] C. S. Tian and Y. R. Shen, Proc. Natl. Acad. Sci. U.S.A. 106, 15148 (2009).

[54] K. Roger and B. Cabane, Angew. Chem., Int. Ed. Engl. 51, 5625 (2012).

[55] Y. Uematsu, D. J. Bonthuis, and R. R. Netz, Langmuir 36, 3645 (2020).

[56] M. S. Barbosa, N. Balke, W.-Y. Tsai, C. Santato, and M. O. Orlandi, J. Phys. Chem. Lett. 11, 3257 (2020).

[57] P. Tarazona, Phys. Rev. Lett. 84, 694 (2000).

[58] J. Hernández-Muñoz, E. Chacón, and P. Tarazona, J. Chem. Phys. 151, 034701 (2019).

[59] P. Tarazona, J. A. Cuesta, and Y. Martínez-Ratón, in Theory Simulation Hard-Sphere Fluids Related Systems (Springer Berlin Heidelberg, Berlin, Heidelberg, 2008), pp. 247-341.

[60] R. García and R. Pérez, Surf. Sci. Rep. 47, 197 (2002).

[61] M. Ricci, W. Trewby, C. Cafolla, and K. Voïtchovsky, Sci. Rep. 7, 43234 (2017).

[62] S. R. Van Lin, K. K. Grotz, I. Siretanu, N. Schwierz, and F. Mugele, Langmuir 35, 5737 (2019).

[63] T. Ichii, S. Ichikawa, Y. Yamada, M. Murata, T. Utsunomiya, and H. Sugimura, Jpn. J. Appl. Phys. 59, SN1003 (2020). 